Review

\title{
Impact of Daylight Saving Time on circadian timing system: An expert statement
}

\author{
Meira e Cruz M. ${ }^{\mathrm{a}, *}$, Miyazawa M. ${ }^{\mathrm{b}}$, Manfredini R. ${ }^{\mathrm{c}}$, Cardinali D. ${ }^{\mathrm{d}}$, Madrid J.A. ${ }^{\mathrm{e}}$, Reiter R. ${ }^{\mathrm{f}}$, \\ Araujo J.F. ${ }^{g}$, Agostinho R. ${ }^{\mathrm{h}}$, Acuña-Castroviejo D. ${ }^{\mathrm{b}}$ \\ a Sleep Unit, Cardiovascular Center, University of Lisbon, Faculty of Medicine, Lisbon, Portugal \\ ${ }^{\mathrm{b}}$ Department of Physiology, Faculty of Medicine, Center of Biomedical Research, Parque Tecnologico de Ciencias de la Salud, University of Granada, Granada, Spain \\ ${ }^{c}$ Department of Medical Sciences, Faculty of Medicine, Surgery and Prevention, University of Ferrara, Ferrara, Italy \\ ${ }^{\mathrm{d}}$ BIOMED-UCA-CONICET, Department of Teaching and Research, Faculty of Medical Sciences, Pontificia Catholic University of Argentina, Buenos Aires, Argentina \\ ${ }^{\mathrm{e}}$ Department of Physiology, Faculty of Biology, University of Murcia, Spain \\ ${ }^{\mathrm{f}}$ Department of Cell Systems and Anatomy, UT Health, United States \\ ${ }^{\mathrm{g}}$ Laboratory of Neurobiology and Biological Rhythmicity, Department of Physiology and Biophysics, Federal University of Rio Grande do Norte, Natal, Brazil \\ ${ }^{\mathrm{h}}$ Institute of Astrophysic and Space Sciences, Department of Physics, Faculty of Sciences, University of Lisbon, Lisbon, Portugal
}

\section{Introduction}

Even though that in the very beginning of Daylight Saving Time (DST) policy, standards were decided individually by each country, and were not regulated, most European member States have progressively been adopting the so called "summer time" since 1970s or 1980s which had been gradually harmonized under EU law [1]. Public discomfort have been argued for years and claims from several social groups regarding the need for a public inquiry were attended. However, after European Union decided to properly ask citizens about their feelings regarding Daylight Saving Time, a small fraction of European citizens (68\% from Germany) had told that they don't want to change the time twice a year. Despite the very statistically biased result, there are still misgivings about the biomedical and clinical meanings of this position. Therefore a significant amount of uncertainty related to the actual impact of DST on health persists both in general public and in the clinical and scientific community. Actually, at the same time discussion seemed ready to be closed in EU, some countries persist with this trend and some others try to implement variants.

\section{The group and the topics on discussion}

With this in mind, an international group of specialists in clinical medicine, physiology and immunology with expertise on chronobiology and sleep medicine (Table 1) joined together in August 2018 with the aims of: 1) to reflect, analyze and clarify on the literature regarding the numerous effects potentially attributed to DST-induced circadian misalignment; 2) to define whether adherence to DST should be considered a dangerous rather risky behavior in terms of health and wellbeing.

\section{World changed since DST was implemented}

Since Benjamin Franklin idea for saving coal with supplementary light in summer [2], world has developed and lamps, TVs, computers and smartphones became available for the majority of the population. Shiftwork and intercontinental travels are no longer rare conditions for a great number of people, and transitions between day and night became less marked by related geophysical factors like sun during daytime and darkness or moon light during nighttime to conform to the law of social rules and " $24 \mathrm{~h}$ open" commercial dictation.

\section{DST - behind sleep}

It is still common that whenever talking about circadian rhythms, people think about sleep. There was 88 titles available in September 2018 on PubMed in answer to the search criteria of DST, most of them made reference to sleep/wake or to rest/activity cycle.

That's actually the most obvious circadian rhythm and that is why it is used as the most common variable to study the impact of circadian disruption. Nevertheless, circadian timing system is a complex physiological network that comprises a hierarchy of peripheral oscillators (peripheral biological clocks) commanded by a central pacemaker, the suprachiasmatic nucleus (master clock). This well-organized and interactive system causes success to have the right time to occur in virtually all biological forms. Not only sleep and wake time, but also meal time, several hormones, blood pressure, the better time for alertness and attention, time for the greater immune protection, and time for being wise as the prefrontal cortex, so relevant for critical judgement, is influenced by circadian regulation.

There are also times of risk to suffer disease that are regulated by circadian control machinery. To complete this multioscillatory system,

\footnotetext{
* Corresponding author.

E-mail address: mcruz@medicina.ulisboa.pt (M. Meira e Cruz).
} 
Table 1

Participants of the consensus group DST-2018.

\begin{tabular}{|c|c|c|}
\hline Name & Afiliation/center & City, Country \\
\hline Miguel Meira e Cruz & Sleep Unit, CCUL - Cardiovascular Center of University of Lisbon, Lisbon School of Medicine & Lisboa, Portugal \\
\hline Maasaki Miyasawa & Department of Immunology, Faculty of Medicine and Anti-Aging Center, Kindai University & Osaka, Japan \\
\hline Roberto Manfredini & Department of Medical Sciences, Faculty of Medicine, Surgery and Prevention, University of Ferrara & Ferrara, Italy \\
\hline Daniel Cardinali & $\begin{array}{l}\text { BIOMED-UCA-CONICET and Department of Teaching and Research, Faculty of Medical Sciences, Pontificia Universidad } \\
\text { Católica Argentina }\end{array}$ & Buenos Aires, Argentina \\
\hline Juan Antonio Madrid & Department of Physiology, Faculty of Biology, University of Murcia & Murcia, Spain \\
\hline Russel Reiter & Department of Cell System and Anatomy, UT Health & Texas, United States \\
\hline John Fontenele Araujo & Laboratory of Neurobiology and Biological Rithmicity, Department of Physiology & Natal, Brasil \\
\hline Rui Agostinho & Institute of Astrophysic and Space Sciences, Department of Physics, Faculty of Sciences, University of Lisbon & Lisboa, Portugal \\
\hline Darío Acuña-Castroviejo & $\begin{array}{l}\text { Department of Physiology, Faculty of Medicine, Center of Biomedical Research, Parque Tecnologico de Ciencias de la Salud, } \\
\text { University of Granada, Granada }\end{array}$ & Granada, Spain \\
\hline
\end{tabular}

thousands of genes organized in clusters peak at different times coping with the different functions of every cell in each moment. Despite all those dynamics occur within the physiological context in a highly synchronized fashion, several time-related disturbances and interferences may plausibly change the multicomponent system dictating internal time [3]. The importance of the complex circadian organization for understanding the mechanism of the disease was stated as a new frontier for medicine by Fred Turek, which used for the first time the term "Circadian Medicine" [4].

\section{DST is different from traveling one hour time zone}

Frequently, the impact of the DST has often been compared with that which occurs when we travel to a time zone that differs by one hour; however, the resynchronization dynamics is quite different in both situations. As predicted by the phase response curve (PRC) to light [5], a phase delay in our sleep time will occur when we increase the light exposure in the afternoon and decrease it in the morning with respect to our subjective internal time (SIT). On the contrary more light in the morning and less in the afternoon will be followed by a phase advance. Applying the PRC to DST, when we travel one hour to the West, we can get up and go to bed one hour later in close association to one hour of delay in the sunrise and sunset respect to SIT. This will promote a phase delay which accelerates our resynchronization; however after the end of DST in October, we will get up and go to bed also an hour later but the sunset and sunrise will continue at the same time with respect to the SIT thus, immediately after the DST, we will expose to more light in the morning and less in the afternoon favoring a phase advance that counteract our rapid resynchronization.

In a similar way, when we travel to the East for one hour (process which can be thought similar to the DST of March) the sunrise and sunset occur one hour earlier with respect to SIT, promoting the phase advance, as is desired; but after the Spring DST, suddenly we pass to receive more light in the afternoon and less in the morning, which will favor the phase delay, again an undesired effect that prolong the resynchronization time. Therefore the effect of the DST is more persistent and is not at all comparable to the effect of traveling one time zone.

\section{Microsystems and rhythmicity}

Genes, molecules, biochemical structures, interactive physiological behaviors and functions may all be affected with an apparently innocuous stimulus such as that determined by changing only one hour during DST. However, clinical consequences range from an absence of perception of any effect to severe discomfort which sometimes may persist until the recovery of the previous schedule [6]. DNA damage repair response that protects our genetic material is itself strongly connected to the biological clock [7] and thus radiation-related cancer induction might be affected by the derangement of circadian timing. Urthermore, immune cells that survey the emergence of cancer cells possess intrinsic biological clocks, and immune mediators including cytokines and chemokines exert strong influence on circadian rhythms even at behavioral level [8]. It has been shown that higher incidence of cancer and autoimmune diseases are caused by circadian disruption.

Also the daily variation in brain neurotransmitters, the activity of the autonomous nervous system, endocrine and metabolic functions are under the control of the $24 \mathrm{~h}$ internal master clock. Cell division is "circadian clock-dependent" suffering consequences when internal time disruptive events occur [9]. Actually, the presence of light-at-night, a chrono disruptive factor in humans, is likely linked to cancer and human cancer cells produce the PASD1 - a protein that silences the circadian clock allowing oncogenic transformation [10].

\section{Individual properties and vulnerabilities}

Capacity of human circadian clock's adjustment to seasonal oscillation was also shown to be disrupted by that DST-related one hour loss in spring which seems to affect later chronotypes in a higher degree when compared to the morning chronotypes [11]. At low latitude locations the uselessness of DST may be even greater since the change in photoperiod near equator is negligible.

Time doesn't go by through a rat as it goes by through a human being. Lack of studies and methodological issues make hard to take definite conclusions regarding the real impact of $1 \mathrm{~h}$ change in biological internal microsystems, but behavioral phenotypes essentially reveal that DST it is not friendly $[6,11]$. The economic argument that the DST reduce electrical energy consumed hasn't had justified discomfort related to DST and actually seems to be unacceptable nowadays $[12,13]$.

\section{Chronodisruption, chronotypes and cardiometabolic risk}

Desynchronization of an organism regarding its environment through circadian clock derangements may lead to the development of cardiovascular diseases [14]. Most cardiovascular functions are organized according to a specific circadian order, characterized by rhythmic, predictable-in-time differences in its physiological status. Thus, human beings are exposed either to rhythmic variations in the susceptibility to morbid and mortal events, and to the ability of temporal changes of cardiovascular unfavourable mechanisms to precipitate and graduate the overt expression of disease. This complex inter-relationship, in which several factors, not harmful if taken alone, may trigger unfavourable events when present together at the same time, gives rise to the concept of "chronorisk" [15].

It is already known that the occurrence of cardiovascular events exhibits an evident morning preference [16], and stress plays crucial role upon the circulatory system particularly in the morning [17]. An association between DST and myocardial infarction (MI) was first observed by Janszky \& Ljung [18], and further confirmed. In particular, a higher frequency of MI onset -ranging from 5 to $25 \%$ - was observed after the spring DST transition, and especially on Monday or in the first days of the week [19]. This Monday preference is not surprising at all, 
since it has been observed for MI, stroke, and even for the stress-induced Takotsubo syndrome [20-22].

However, some important information to interpret these data, such as the possible impact of sex and individual circadian preference (chronotype), is lacking. A possible higher frequency of infarctions in women, as observed by Janszky \& Ljung [18], lack to be confirmed [4] and chronotype-related associations should be further tested [19].

Chronotype, which refers to the individual preference regarding daily activity patterns and sleep wake cycles differ with regards to their sleeping behavior, personality, mental health, smoking and dietary habits, school achievements, and so on [23]. Individuals with late chronotype more frequently show increased vulnerability to psychological distress [24] and sleep-related issues, e.g., later bedtime and wake-up, decreased sleep quality and quantity, more pronounced metabolic risk, reduced physical activity, and unhealthy dietary patterns, including also smoking and drinking habits [25,26], Moreover, late chronotype seem to have more problems in adjusting to the spring shift of DST [27].

\section{Lack of an intregrative approach}

Studies rejecting the influence of DST on circadian physiology are failing to integrate the scientific basic and clinical knowledge. The human body is constituted by 30 billions of cells; each cell contains their own clock that regulates its daily functions, and these clocks are exquisitely synchronized by the master clock. On the other hand, the human gut have more than 40 thousand millions of bacteria which are a key part of the brain-gut axis maintaining the body balance and the functions of mucosal immune system that constitutes the largest number of body's immune cells. Each one these bacteria have its own clock influenced also by the suprachiasmatic nucleus.

\section{Conclusion}

Impact of DST over suprachiasmatic master clock and then, over peripheral oscillators seems likely to be sufficient to cause an internal chronodisruption leading, at least theoretically, to significant impairments of homeostasis, at the cellular level, tissue and organ levels and body/systemic level favoring disease. Accepting that science is dynamic and further clarifications are needed should not allow to neglect the potential harm that actual knowledge suggest.

Accordingly the first version of the consensus regarding Impact of DST on Circadian Timming System authors concluded that: 1. Although exact mechanisms are not assured at this moment for all related genetic, molecular and neurobiological processes, there is sufficient literature showing the adverse impact of the DST on different levels of circadian timing system, either related to circadian master clock and multiple peripheral oscillators from which depends adequate organic function; 2 . since there is evidence on the potentially negative effects of DST-related disruption of circadian timing system associated to several negative health outcomes, DST cannot be encouraged and therefore should be discontinued.

\section{Conflict of interest}

All authors declare that dont have any conflict of interest regarding the present study.

\section{References}

[1] Anglmayer Irmgard. EU summer-time arrangements under Directice 2000/84/EC Ex-post impact assessment. October 2017.

[2] The Ingenious Dr. Franklin. Goodman Nathan G, editor. Selected Scientific Letters. University of Pennsylvania Press; 1931. p. 17-22.

[3] Meira e Cruz M, Acuña-Castroviejo D. Intern Emerg Med 2018 Aug 27. https://doi. org/10.1007/s11739-018-1934-7. [Epub ahead of print].

[4] Turek FW. Circadian clocks: not your grandfather's clock. Science 2016 Nov 25;354(6315):992-3.

[5] Khalsa SB, Jewett ME, Cajochen C, Czeisler CA. A phase response curve to single bright light pulses in human subjects. J Physiol 2003;549:945-52.

[6] Alencar JCN, Leocadio-Miguel MA, Duarte LL, Louzada F, Fontenele Araujo J, Pedrazzoli M. Self-reported discomfort associated with Daylight Saving Time in Brazilian tropical and subtropical zones. Ann Hum Biol 2017;44(7):628-35.

[7] Jackson SP, Bartek J. The DNA-damage response in human biology and dissdeaee. Nature 2009;461:1071-8.

[8] Cermakian N, et al. Crosstalk between the circadian clock circuitry and the immune system. J Biol Med Rhythm Res 2013;30:870-88.

[9] Shostak A, Clock Circadian. Cell division, and cancer: from molecules to organisms. Int J Mol Sci 2017 Apr;18(4):873.

[10] Michael AK, et al. Cancer/testis antigen PASD1 silences the circadian clock. Mol Cell 2015;58:743-54.

[11] Kantermann T, Juda M, Merrow M, Roenneberg T. The human circadian clock's adjustement is disrupted by daylight saving time. Curr Biol 2007;17(22):1996-2000.

[12] Shaffer B. Location matters: daylight saving time and electricity use. MPRA Paper. 2017. (RePEc:pra:mprapa:84053).

[13] Sarkar J, Dullek U, Torgler B, Schaffner M. The implications of daylight saving time: A quasi-natural experiment on cognitive performance and risk taking behaviour. Economic Modelling. 2018. p. 390-400. RePEc:eee:ecmode:v:70:y:2018:i:c:p.

[14] Young ME, Phill D. Circadian control of cardiac metabolism: phsysiologic roles and pathologic implications. Methodist Debakey Cardiovasc J 2017 JanMar;13(1):15-9.

[15] Portaluppi F, Manfredini R, Fersini C. From a static to a dynamic concept of risk: the circadian epidemiology of cardiovascular events. Chronobiol Int 1999;16(1):33-49.

[16] Smolensky MH, Portaluppi F, Manfredini R, Hermida RC, Tiseo R, Sackett-Lundeen LL, et al. Diurnal and twenty-four hour patterning of human diseases: cardiac, vascular, and respiratory diseases, conditions, and syndromes. Sleep Med Rev 2015;21:3-11.

[17] Manfredini R, Boari B, Salmi R, Malagoni AM, Manfredini F. Circadian rhythm effects on cardiovascular and other stress-related events. In: Fink G, editor. Encyclopedia of stress. Second EditionOxford: Academic Press; 2007. p. 500-5.

[18] Janszky I, Ljung R. Shift to and from daylight saving time and incidence of myocardial infarction. N Engl J Med 2008;359(18):1966-8.

[19] Manfredini R, Fabbian F, De Giorgi A, Zucchi B, Cappadona R, Signani F, et al. Daylight saving time and myocardial infarction: should we be worried? Eur Rey Med Pharmacol Sci 2018;22(3):750-5.

[20] Gnecchi-Ruscone T, Piccaluga E, Guzzetti S, Contini M, Montano N, Nicolis E. Mornings and Monday: critical periods for the onset of acute myocardial infarction. Eur Heart J 1994;15(7):882-7.

[21] Manfredini R, Casetta I, Paolino E, la Cecilia O, Boari B, Fallica E, et al. Monday preference in onset of ischemic stroke. Am J Med 2001;111(5):401-3.

[22] Manfredini R, Citro R, Previtali M, Vriz O, Ciampi Q, Pascotto M, et al. Bossone E; Takotsubo Italian Network investigators. Monday preference in onset of takotsubo cardiomyopathy. Am J Emerg Med 2010;28(6):715-9.

[23] Kivelä Liia, Papadopoulos Marinos Rodolfos, Antypa Niki. Chronotype and Psychiatric Disorders. Curr Sleep Med Rep 2018;4(2):94-103.

[24] Roeser K, Obergfell F, Meule A, Vogele C, Schlarb AA, Kubler A. Of larks and hearts -morningness/eveningness, heart rate variability, and cardiovascular stress response at different times of day. Physiol Behav 2012;106(2):151-7.

[25] Lucassen EA, Zhao X, Rother KI, Mattingly MS, Courville AB, de Jonge L, et al. Sleep Extension Study Group. Evening chronotype is associated with changes in eating behavior, more sleep apnea, and increased stress hormones in short sleeping obese individuals. PLoS One 2013;8(5):e56519.

[26] Fabbian F, Zucchi B, De Giorgi A, Tiseo R, Boari B, Salmi R, et al. Chronotype, gender and general health. Chronobiol Int 2016;33(7):863-82.

[27] Harrison Y. The impact of daylight saving time on sleep and related behaviours. Sleep Med Rev 2013;17(4):285-92. 